\title{
Antibacterial Properties of Titanate Nanofiber Thin Films Formed on a Titanium Plate
}

\author{
Mitsunori Yada, ${ }^{1}$ Yuko Inoue, ${ }^{1}$ Iwao Noda, ${ }^{2}$ Tomohiro Morita, ${ }^{1}$ Toshio Torikai, ${ }^{1}$ \\ Takanori Watari, ${ }^{1}$ and Takao Hotokebuchi ${ }^{3}$ \\ ${ }^{1}$ Department of Chemistry and Applied Chemistry, Faculty of Science and Engineering, Saga University, 1 Honjo, \\ Saga 840-8502, Japan \\ ${ }^{2}$ Japan Medical Materials Corporation, Uemura Nissei Building 9F 3-3-31 Miyahara, Yodogawa-ku, Osaka 532-0003, Japan \\ ${ }^{3}$ Department of Orthopaedic Surgery, Faculty of Medicine, Saga University, 5 Nabeshima, Saga 849-8501, Japan \\ Correspondence should be addressed to Mitsunori Yada; yada@cc.saga-u.ac.jp
}

Received 16 January 2012; Accepted 25 September 2012

Academic Editor: Gajanan S. Bhat

Copyright (c) 2013 Mitsunori Yada et al. This is an open access article distributed under the Creative Commons Attribution License, which permits unrestricted use, distribution, and reproduction in any medium, provided the original work is properly cited.

\begin{abstract}
A sodium titanate nanofiber thin film and a silver nanoparticle/silver titanate nanofiber thin film formed on the surface of a titanium plate exhibited strong antibacterial activities against methicillin-resistant Staphylococcus aureus, which is one of the major bacteria causing in-hospital infections. Exposure of the sodium titanate nanofiber thin film to ultraviolet rays generated a high antibacterial activity due to photocatalysis and the sodium titanate nanofiber thin film immediately after its synthesis possessed a high antibacterial activity even without exposure to ultraviolet rays. Elution of silver from the silver nanoparticle/silver titanate nanofiber thin film caused by the silver ion exchange reaction was considered to contribute substantially to the strong antibacterial activity. The titanate nanofiber thin films adhered firmly to titanium. Therefore, these titanate nanofiber thin film/titanium composites will be extremely useful as implant materials that have excellent antibacterial activities.
\end{abstract}

\section{Introduction}

Sodium titanate thin films with various nanostructures are known to form on titanium surfaces when titanium metal is soaked in a $\mathrm{NaOH}$ solution [1-5]. Since these thin films possess excellent mechanical properties derived from titanium and an outstanding ability to form apatite (i.e., osteoconductivity) derived from sodium titanate, they have been widely studied as biocompatible materials for use in artificial joints [6-15]. Recently, many studies have focused on making Tibased antibacterial materials in order to prevent infection during and after an artificial joint replacement surgery [16]. Silver has been particularly well studied as an antibacterial substance that can prevent development of drug-resistant bacteria while maintaining biological safety $[17-22]$. There have been reports of the use of alloying [17], magnetron cosputtering [18], anodization and electrodeposition [19], microarc oxidation [20], thermal spraying [21], and plasma electrolytic oxidation [22].
Our group found that a sodium titanate nanotube thin film could be formed on the surface of a titanium plate after hydrothermal treatment of a titanium plate in a $10 \mathrm{~mol} / \mathrm{L}$ $\mathrm{NaOH}$ solution, followed by a fixing treatment [23]. Performing a silver ion exchange treatment on the formed thin film using a silver acetate solution transformed the sodium titanate nanotube thin film into a silver nanoparticle/silver titanate nanotube thin film. The obtained thin film possessed an excellent antibacterial activity and apatite forming ability $[24,25]$.

In the present paper, we introduce a sodium titanate nanofiber thin film. The sodium titanate nanofiber thin film was obtained using a $4 \mathrm{~mol} / \mathrm{L} \mathrm{NaOH}$ solution instead of a $10 \mathrm{~mol} / \mathrm{L} \mathrm{NaOH}$ solution. In the synthesis of the sodium titanate nanotube thin film, when the sample was washed with water to remove excess $\mathrm{NaOH}$ immediately after the hydrothermal treatment, the nanotube thin film was detached from the titanium plate. Therefore, fixing treatment was required. On the other hand, the sodium titanate nanofiber 
thin film firmly adhered to the surface of a titanium plate immediately after performing the hydrothermal treatment and therefore required no special fixing treatment. The use of titanate thin film/titanium composites as biocompatible materials requires firm adherence between the thin film and titanium. Therefore, the nanofiber thin film, which has excellent adherence to titanium, appears to be a promising biocompatible material. However, only a single study [4] has investigated the antibacterial activity against Escherichia coli of titanate nanofiber thin films due to photocatalysis. Further detail is still needed regarding antibacterial activity. In the present study, a sodium titanate nanofiber thin film was formed on the surface of a titanium plate by hydrothermal treatment of the plate in a $\mathrm{NaOH}$ solution. The obtained thin film was then transformed into a silver nanoparticle/silver titanate nanofiber thin film by soaking it in a silver acetate solution. Methicillin-resistant Staphylococcus aureus (MRSA), which is one of the major bacteria causing inhospital infections, was then used to evaluate the antibacterial activity of the sodium titanate nanofiber thin film due to photocatalysis and the antibacterial activity of the silver nanoparticle/silver titanate nanofiber thin film due to elution of silver ions.

\section{Experimental Section}

2.1. Syntheses of a Sodium Titanate Nanofiber Thin Film and a Silver Nanoparticle/Silver Titanate Nanofiber Thin Film. A titanium plate $(20 \times 20 \times 2 \mathrm{~mm})$ was reacted hydrothermally with $20 \mathrm{~mL}$ of a $4 \mathrm{~mol} / \mathrm{L} \mathrm{NaOH}$ solution at $160^{\circ} \mathrm{C}$ for $20 \mathrm{~h}$. After the hydrothermal treatment, the obtained sample was repeatedly washed with water, resulting in a titanium plate coated with a sodium titanate nanofiber thin film. The plate was then soaked in $12 \mathrm{~mL}$ of a $0.05 \mathrm{~mol} / \mathrm{L}$ silver acetate solution for $3 \mathrm{~h}$ at $40^{\circ} \mathrm{C}$, repeatedly washed with distilled water, and dried in a dark place to transform the sodium titanate nanofiber thin film into a silver nanoparticle/silver titanate nanofiber thin film. The antibacterial activity was evaluated by synthesizing a sodium titanate nanofiber thin film and a silver nanoparticle/silver titanate nanofiber thin film on a titanium plate $(50 \times 50 \times 2 \mathrm{~mm})$ using solutions with the same concentration but different volumes. Transmission electron microscopy (TEM) was performed with a Hitachi $\mathrm{H}-800 \mathrm{MU}$ instrument and a JEOL JEM-1210 instrument. A sample for TEM observation was prepared by sprinkling powders obtained by scaling the thin film onto a standard TEM grid. The acceleration voltage for TEM observation was $120 \mathrm{kV}$. Scanning electron microscopy (SEM) was performed with a Hitachi S-3000N. Energy-dispersive X-ray microanalysis (EDX) was performed with an EDAX Genesis 2000 instrument. X-ray diffraction (XRD) measurements were performed with a Shimadzu XRD-6100 instrument using Cu $\mathrm{K} \alpha$ radiation.

2.2. Antibacterial Activity Evaluation of the Thin Film. The antibacterial activity was evaluated using partially modified Japanese Industrial Standard test (JIS Z2801). The samples were disinfected with alcohol for $10 \mathrm{~min}$ and dried in air at $25^{\circ} \mathrm{C}$ for $18 \mathrm{~h}$. A sterilized polyethylene film was used as a blank sample. The environment of infection in an actual living body was mimicked by using a inactivated bovine serum as the solvent for the bacterial suspension, which reproduced eutrophic conditions, and the antibacterial activity was evaluated against MRSA that possessed the biofilm formation gene.

A $0.2 \mathrm{~mL}$ volume of the bacterial suspension was dropped onto the sample plate $(50 \times 50 \times 2 \mathrm{~mm})$, a $40 \times 40 \mathrm{~mm}$ polyethylene film (Elmex Corp.) was placed on the plate, and the sample plate was cultured at $37^{\circ} \mathrm{C}$ for $24 \mathrm{~h}$. Subsequently, the number of viable bacteria was counted. The same experiment was partially performed using a different sample plate $(20 \times 20 \times 2 \mathrm{~mm})$, a different quantity of the bacterial suspension, and a different film size. Each of the thin films (the sodium titanate nanofiber thin film, the silver nanoparticle/silver titanate nanofiber thin film, and the sterilized polyethylene film) was evaluated three times, and the average number of viable bacteria was calculated. The average number of viable bacteria was used to calculate the antibacterial activity value. The antibacterial activity value $(R)$ for the sample was calculated as follows:

$$
R=\left\{\log \left(\frac{B}{A}\right)-\log \left(\frac{C}{A}\right)\right\}=\log \left(\frac{B}{C}\right) .
$$

Here, $A, B$, and $C$ are the average viable MRSA counts just after inoculation, after $24 \mathrm{~h}$ for a blank and after $24 \mathrm{~h}$ for a sample, respectively. The antibacterial characteristics due to ultraviolet (UV) irradiation were evaluated using a UV irradiation apparatus that was vertically adjusted to set the illumination as 52-92 Lux and the intensity as $133-236 \mu \mathrm{w} / \mathrm{cm}^{2}$. The UV irradiation was continued for $24 \mathrm{~h}$. The sample plate was disinfected with alcohol and washed with water between each evaluation.

\subsection{Elution of Silver from the Silver Nanoparticle/Silver} Titanate Nanofiber Thin Film. The silver nanoparticle/silver titanate nanofiber thin film formed on a titanium plate $(20$ $\times 20 \times 2 \mathrm{~mm}$ ) was then soaked in $15 \mathrm{~mL}$ of a fetal bovine serum solution at $37^{\circ} \mathrm{C}$, and then left to stand for $24 \mathrm{~h}$. Fetal bovine serum was used to mimic MRSA environment in order to study the elution behavior of silver [24]. The eluate was collected, centrifuged, and filtrated through a $0.22 \mu \mathrm{m}$ filter. The Ag concentration in the eluate was measured by inductively coupled plasma (ICP) mass spectroscopy performed with a Seiko SPQ-8000. This experiment was repeated 10 times, and the Ag concentration in the eluate was measured each time.

\section{Results and Discussion}

3.1. Synthesis of a Sodium Titanate Nanofiber Thin Film and Its Transformation into a Silver Nanoparticle/Silver Titanate Nanofiber Thin Film by a Silver Ion Exchange Treatment. Figure 1(a) (inset) shows a digital photograph of the synthesized thin film, which appeared as a uniform thin film formed on the titanium plate. A sodium titanate nanotube thin film 

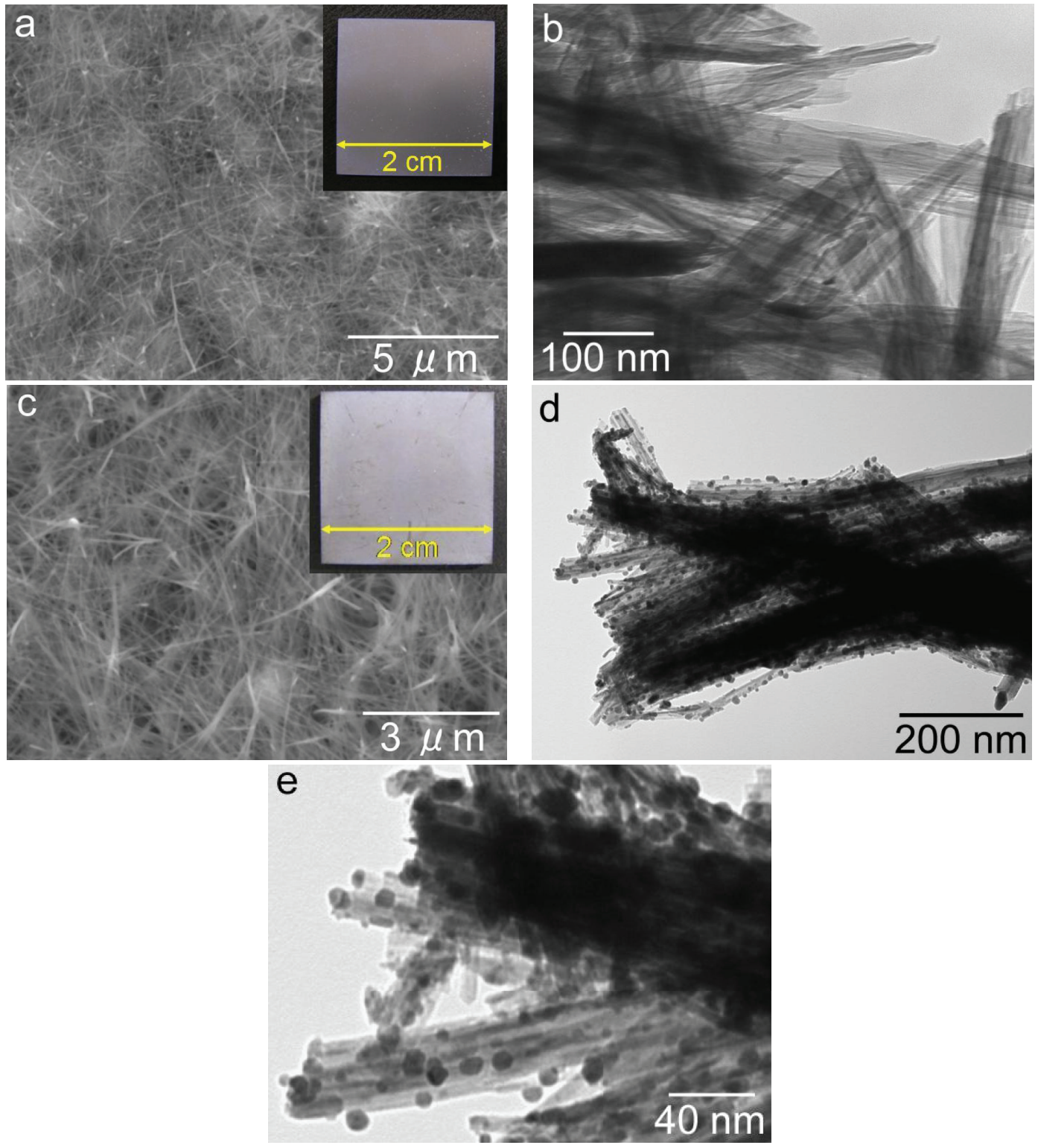

Figure 1: SEM images (a), (c) and TEM images (b), (d), and (e) of the sodium titanate nanofiber thin film (a), (b) and the silver nanoparticle/silver titanate nanofiber thin film (c), (d), and (e). Insets are photographs taken with a digital camera.

exfoliated from the titanium plate when the thin film was washed with a large amount of water immediately after the hydrothermal treatment [23]. In contrast, no exfoliation of the thin film was observed in Figure 1(a) (inset); that is, the thin film firmly adhered to the titanium plate. SEM image (Figure 1(a)) confirmed that the thin film was composed of nanofibers. Peng and Chen [3], Dong et al. [4], and Chi et al. [26] also reported formation of similar one dimensional sodium titanate thin films, such as sodium titanate nanowire thin films $[3,4,26]$ and sodium titanate nanobelt thin film [26], although they used different concentrations of $\mathrm{NaOH}$ solution at different reaction temperatures. The concentration of the $\mathrm{NaOH}$ solution and the reaction temperature were considered to have substantial effects on the structure and the rate of formation of the precursor of sodium titanate and on the morphology of sodium titanate that was finally formed. TEM image (Figure 1(b)) also revealed that the thin film was composed of nanofibers with widths between tens of nm and a little more than $100 \mathrm{~nm}$, and lengths of several $\mu \mathrm{m}$. Cross sections of the thin film indicated a film thickness of approximately $6 \mu \mathrm{m}$.

The XRD pattern of the thin film (Figure 2(a)) showed diffraction peaks attributable to titanate with a layered structure. EDX analysis indicated a molar ratio of $\mathrm{Na}: \mathrm{Ti}: \mathrm{O}=$ $1: 3.04: 6.08$. Sodium titanates with various compositions have been reported previously. Among the reported sodium titanates, the EDX data from the present study most closely agreed with a molar ratio of $\mathrm{Na}_{2} \mathrm{Ti}_{6} \mathrm{O}_{13}$. Therefore, a $\mathrm{Na}_{2} \mathrm{Ti}_{6} \mathrm{O}_{13}$ nanofiber thin film was considered to have formed in the present study. Although their synthesis 


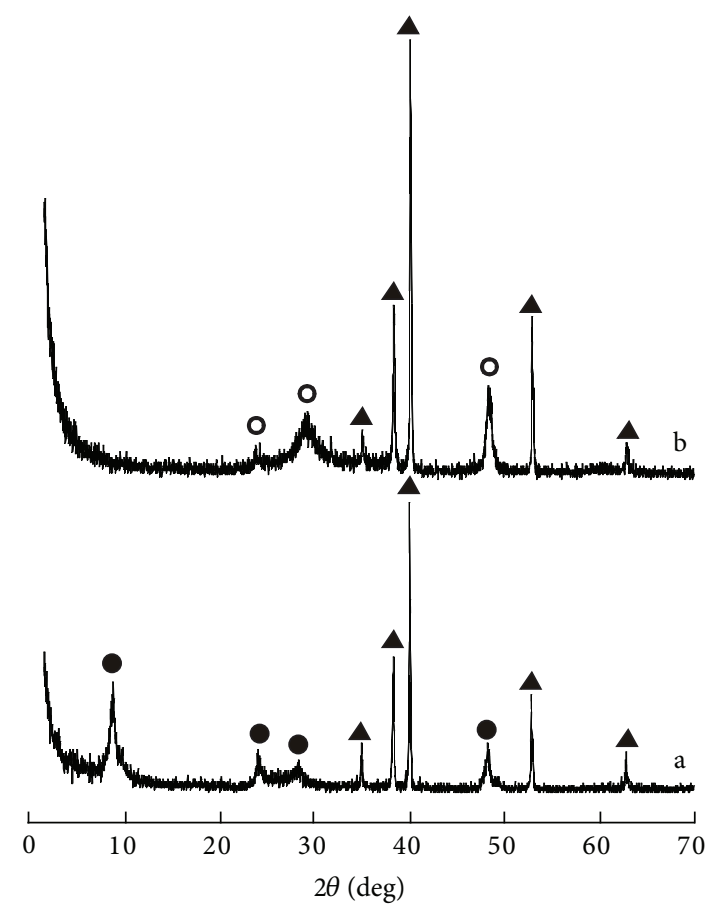

FIGURE 2: XRD patterns of the thin film before and after the silver ion exchange treatment; the XRD pattern immediately after the hydrothermal reaction and prior to the silver ion exchange treatment (a) and the XRD pattern following the silver ion exchange treatment (b). Peak assignment: $\bullet$, sodium titanate; $\mathbf{\Delta}$, titanium; $\bigcirc$, silver titanate.

conditions were different, Peng and Chen [3] reported the synthesis of a $\mathrm{Na}_{2} \mathrm{Ti}_{6} \mathrm{O}_{13}$ nanofiber thin film. Chi et al. [26] also suggested that a $\mathrm{Na}_{2} \mathrm{Ti}_{6} \mathrm{O}_{13}$ nanofiber thin film had been synthesized.

Figure 1(c) (inset) shows the thin film firmly adhered to the titanium plate following the silver ion exchange treatment. The SEM image in Figure 1(c) shows that no large morphological changes at the micrometer level occurred following the silver ion exchange treatment. However, the TEM image (Figure 1(d)) shows the presence of many nanoparticles, between several $\mathrm{nm}$ and a little more than $10 \mathrm{~nm}$ in size, deposited on the surfaces of nanofibers. Figure 1(e), an enlarged image of Figure 1(d), shows that angular polyhedral nanoparticles appear to have formed. These nanoparticles were considered to be composed of silver, and were thought to be deposited due to photocatalysis of titanate. As mentioned below, sodium titanate nanofibers possess a photocatalytic capability. Silver ions in silver titanate were reduced to silver by electrons excited by light irradiation. The formed silver, which was electrically neutral, could not remain within the titanate nanofiber, and silver nanoparticles formed by shifting and aggregating on the surface of the nanofiber. When the thin film was continuously irradiated with light after performing a silver ion exchange treatment, the color of the sample changed to black. However, when the thin film was covered with aluminum foil and stored after performing a silver ion exchange treatment, the color of the sample changed very little. Therefore, the formation of silver nanoparticles was clearly a result of light irradiation, and the mechanism suggested above appeared to explain the formation of these nanoparticles. Since the surfaces of the formed silver nanoparticles were not protected with surfactants or similar compounds, these surfaces might be oxidized or hydroxylated in the solution. The EDX analysis revealed $\mathrm{Ag}$ but no $\mathrm{Na}$ in the sample after the silver ion exchange treatment, indicating that an exchange of $\mathrm{Na}^{+}$ ions for $\mathrm{Ag}^{+}$ions had taken place in the sodium titanate nanofibers. The molar ratio of $\mathrm{Ag} / \mathrm{Ti}$ was 0.638 , which was larger than the original $\mathrm{Na} / \mathrm{Ti}=0.329$ in the sample before the silver ion exchange treatment. The reasons for this were probably that $\mathrm{Ag}^{+}$ions were inserted into titanate following the ion-exchange reaction and silver nanoparticles were deposited onto the surfaces of the nanofibers.

The XRD pattern of the sample after the silver ion exchange treatment (Figure 2(b)) showed the disappearance of the diffraction peak at $2 \theta=8.78^{\circ}$ that was attributed to the sodium titanate layered structure observed in the sample before the silver ion exchange treatment. The diffraction peaks near $2 \theta=24.06^{\circ}$ and $28.22^{\circ}$ attributed to the crystal structure of sodium titanate also became indistinct, while a broad diffraction peak with high intensity appeared near $2 \theta=29.3^{\circ}$, and the intensity of a diffraction peak near $2 \theta=$ $48.3^{\circ}$ increased. These results indicated that the exchange of $\mathrm{Na}^{+}$ions with $\mathrm{Ag}^{+}$ions that took place in the sodium titanate nanofibers changed the crystal structure of titanate from the layered structure to a three-dimensional structure, while maintaining the shape of nanofiber, and consequently, silver titanate nanofibers were formed. A change in the crystal structure was also observed following the silver ion exchange treatment due to the transformation of the sodium titanate nanotube into the silver titanate nanotube [24, 27]. Diffraction peaks attributed to silver were not observed. The reasons for this were considered to be that the diameter of the silver nanoparticles and the amounts of deposited silver nanoparticles were too small to detect.

\subsection{Antibacterial Activity of the Sodium Titanate Nanofiber} Thin Film against MRSA due to Photocatalysis. Table 1 shows the results of evaluation of the sodium titanate nanofiber thin film for antibacterial activity due to photocatalysis. The first evaluation under UV irradiation showed that $3.0 \times$ $10^{5} \mathrm{CFU} /$ sample viable bacteria were present immediately after inoculation of MRSA. At $24 \mathrm{~h}$ after the inoculation, the number of viable bacteria increased to $6.6 \times 10^{8} \mathrm{CFU} / \mathrm{sample}$ on the blank sample but this number decreased to $7.2 \times$ $10^{2} \mathrm{CFU} /$ sample on the sodium titanate nanofiber thin film. These results indicated that MRSA was not killed by UV irradiation alone but was killed by exposing the sodium titanate nanofiber thin film to UV rays. The antibacterial activity value of the sodium titanate nanofiber thin film was calculated to be as high as 5.9. In general, an antibacterial activity value of 2 or higher is considered to represent antibacterial activity. Therefore, the sodium titanate nanofiber thin film possessed a high antibacterial activity under UV irradiation. 


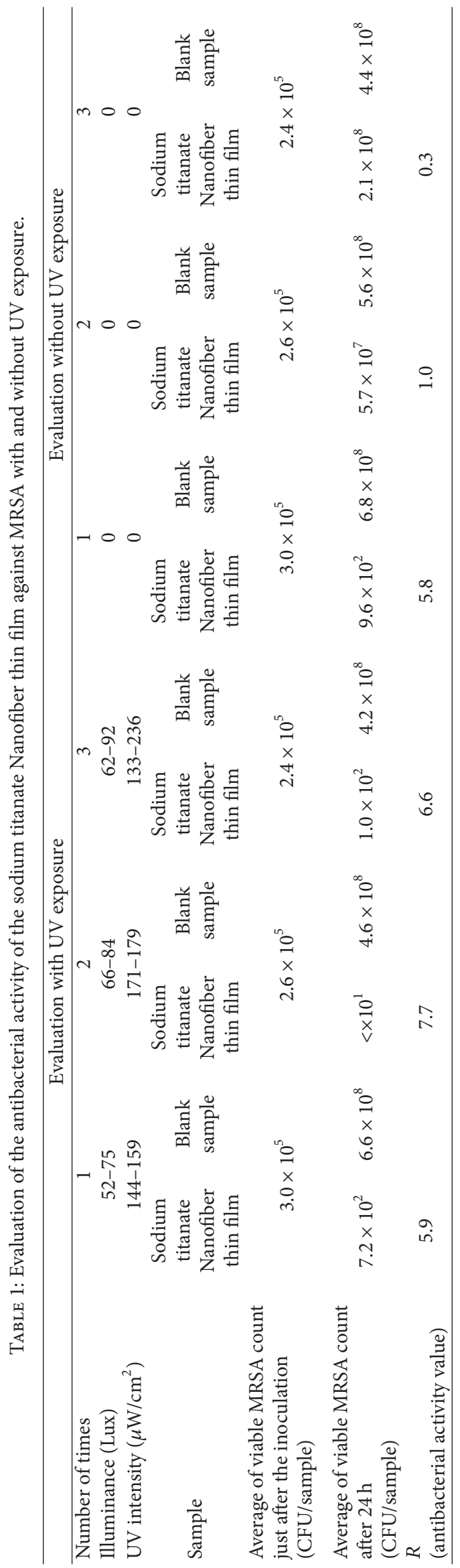


TABLE 2: Evaluation of the antibacterial activity of the sodium titanate nanofiber thin film against E. coli and S. aureus.

\begin{tabular}{lcccc}
\hline Name of bacteria & Sample & $\begin{array}{c}\text { Average of viable bacteria } \\
\text { just after the inoculation } \\
\text { (CFU/sample) }\end{array}$ & $\begin{array}{c}\text { Average of viable bacteria } \\
\text { count after 24h } \\
\text { (CFU/sample) }\end{array}$ & $\begin{array}{c}R \\
\text { (antibacterial activity value) }\end{array}$ \\
\hline Escherichia coli & $\begin{array}{c}\text { Sodium titanate } \\
\text { Nanofiber thin film } \\
\text { Blank sample }\end{array}$ & $2.2 \times 10^{5}$ & $2.7 \times 10^{3}$ & $7.1 \times 10^{8}$ \\
\hline \multirow{2}{*}{ Staphylococcus aureus } & $\begin{array}{c}\text { Sodium titanate } \\
\text { Nanofiber thin film } \\
\text { Blank sample }\end{array}$ & $2.5 \times 10^{5}$ & $2.3 \times 10^{3}$ \\
\hline
\end{tabular}

TABLE 3: Evaluation of the antibacterial activity against MRSA of sodium titanate nanofiber thin films synthesized following different reaction times.

\begin{tabular}{|c|c|c|c|c|}
\hline Reaction time & Sample & $\begin{array}{c}\text { Average of viable MRSA count } \\
\text { just after the inoculation } \\
\text { (CFU/sample) }\end{array}$ & $\begin{array}{c}\text { Average of viable MRSA count } \\
\text { after } 24 \mathrm{~h} \\
(\mathrm{CFU} / \text { sample })\end{array}$ & $\begin{array}{c}R \\
\text { (antibacterial activity value) }\end{array}$ \\
\hline \multirow[t]{2}{*}{1} & $\begin{array}{c}\text { Sodium titanate } \\
\text { Nanofiber thin film }\end{array}$ & $2.8 \times 10^{5}$ & $4.3 \times 10^{7}$ & 1.2 \\
\hline & Blank sample & & $6.5 \times 10^{8}$ & \\
\hline \multirow[t]{2}{*}{5} & $\begin{array}{c}\text { Sodium titanate } \\
\text { Nanofiber thin film }\end{array}$ & $2.6 \times 10^{5}$ & $3.9 \times 10^{8}$ & 0.3 \\
\hline & Blank sample & & $7.9 \times 10^{8}$ & \\
\hline \multirow[t]{2}{*}{12} & $\begin{array}{c}\text { Sodium titanate } \\
\text { Nanofiber thin film }\end{array}$ & $2.6 \times 10^{5}$ & $6.5 \times 10^{5}$ & 3.1 \\
\hline & Blank sample & & $7.9 \times 10^{8}$ & \\
\hline \multirow[t]{2}{*}{20} & $\begin{array}{c}\text { Sodium titanate } \\
\text { Nanofiber thin film }\end{array}$ & $2.1 \times 10^{5}$ & $2.1 \times 10^{2}$ & 6.5 \\
\hline & Blank sample & & $6.0 \times 10^{8}$ & \\
\hline
\end{tabular}

The antibacterial activity of the sodium titanate nanofiber thin film was also evaluated without exposure to UV rays (i.e., in the dark). Table 1 shows a markedly high antibacterial activity value of 5.8. The same sample was repeatedly evaluated for antibacterial activity with UV exposure and showed antibacterial activity values as high as 7.7 and 6.6 for the second and third evaluations, respectively. The antibacterial activity values without UV exposure, for the second and third evaluations, were as low as 1.0 and 0.3 , respectively. Therefore, the antibacterial activity began to decline at the second evaluation and afterward.

In summary: (1) exposure of the sodium titanate nanofiber thin film to UV rays generated a high antibacterial activity due to photocatalysis; and (2) the sodium titanate nanofiber thin film immediately after its synthesis possessed a high antibacterial activity even without exposure to UV rays. Dong et al. [4] previously reported the antibacterial activity of a titanate nanofiber thin film due to photocatalysis. However, the composition of the thin film was not documented and only the antibacterial activity against gram-negative Escherichia coli was investigated. In the present study, the composition of sodium titanate was first elucidated and its antibacterial activity was evaluated against gram-positive MRSA, which actually causes inhospital infections. Therefore, the present study adds new information to the results reported by Dong et al. [4] and provides a more in-depth understanding of the antibacterial activity of the titanate nanofiber thin film due to photocatalysis.

Our group has previously reported that the antibacterial activity value of a sodium titanate nanotube thin film without exposure to UV rays was 1.7 , and that of a sodium titanate thin film with a porous network structure was 1.8 [24]. A sodium titanate nanosheet thin film was hydrothermally synthesized using a $1 \mathrm{~mol} / \mathrm{L} \mathrm{NaOH}$ solution at $160^{\circ} \mathrm{C}$ for $20 \mathrm{~h}$. The antibacterial activity value of this synthesized thin film was 2.1. These results indicated that the sodium titanate nanofiber thin film alone had a markedly high antibacterial activity. As shown in Table 2, the antibacterial activity values of the sodium titanate nanofiber thin film against gramnegative E. coli and gram-positive Staphylococcus aureus, evaluated without UV exposure, were 5.5 and 4.7, respectively. This indicated that the sodium titanate nanofiber thin film had high antibacterial activity against different bacteria. The reproducibility of the antibacterial activity evaluation against MRSA, without UV exposure, was confirmed by investigating the antibacterial activities of sodium titanate nanofiber thin films that were synthesized following different reaction times. The SEM images in Figure 3 show that a nanofiber thin film was clearly formed as the reaction time proceeded. The XRD pattern in Figure 4 shows that the intensity of each diffraction peak or the crystallinity increased 

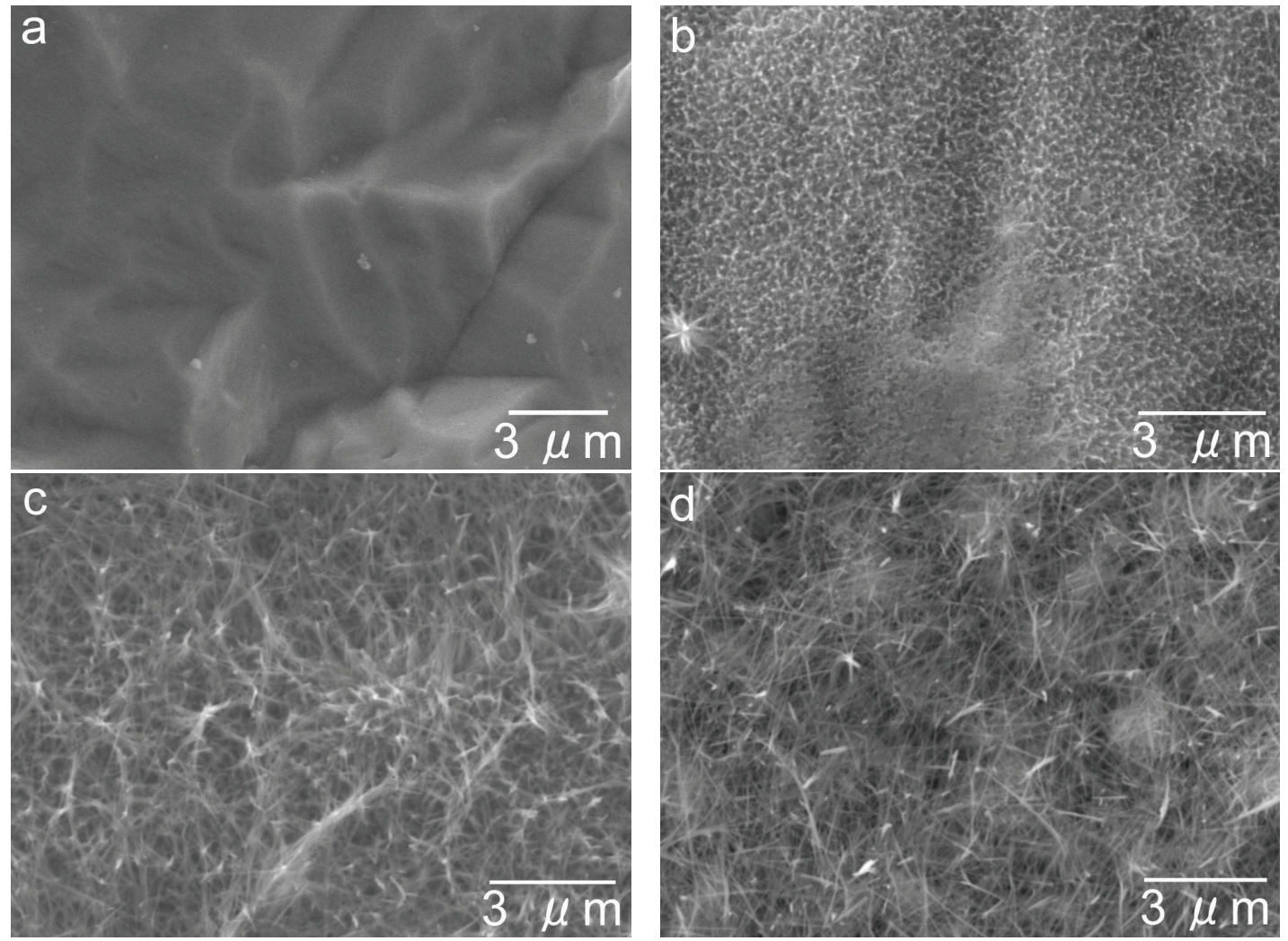

FIGURE 3: SEM images of sodium titanate nanofiber thin films synthesized following different reaction times; the titanium plate before the

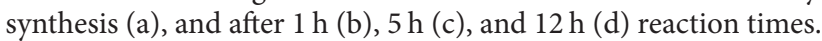

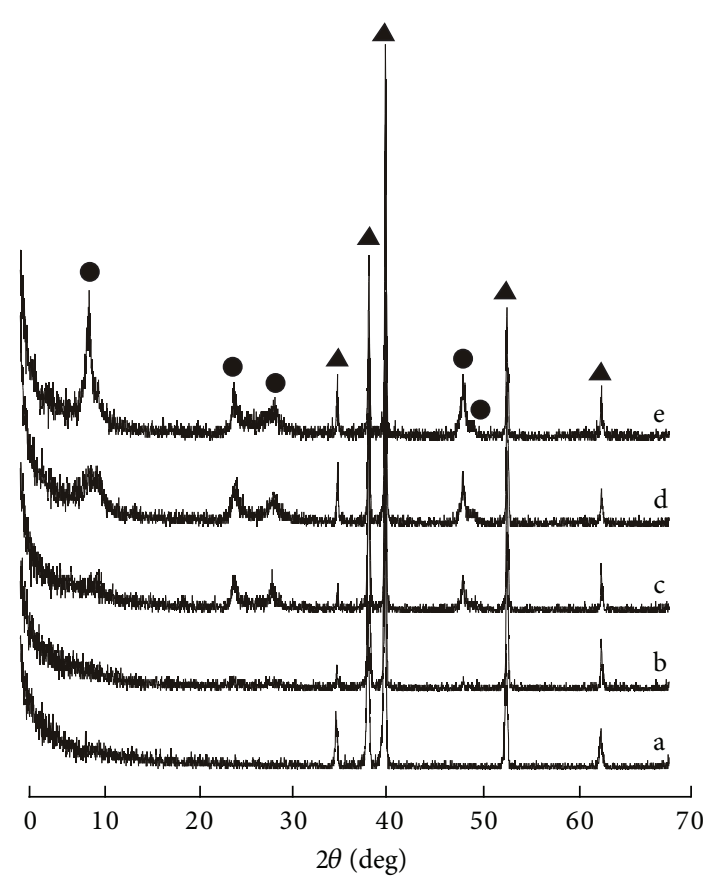

FIGURE 4: XRD patterns of sodium titanate nanofiber thin films following different reaction times; the titanium plate before the synthesis (a), and after $1 \mathrm{~h} \mathrm{(b),} 5 \mathrm{~h} \mathrm{(c),} 12 \mathrm{~h}$ (d), and $20 \mathrm{~h}$ (e) reaction times. Peak assignment: $\bullet$, sodium titanate; $\mathbf{\wedge}$, titanium. as the reaction time proceeded. Table 3 shows that the antibacterial activity values of thin films synthesized with the reaction times of $1 \mathrm{~h}$ and $5 \mathrm{~h}$ were below 2 . Therefore, these films had no clear antibacterial activity. However, extending the reaction time to $12 \mathrm{~h}$ resulted in an antibacterial activity value of 3.1. When the reaction time was $20 \mathrm{~h}$, the value was 6.5. Therefore, increases in the crystallinity of the nanofibers were accompanied by increases in the antibacterial activity of the thin film. At this moment, we cannot clearly explain the reason for these high antibacterial activity values without UV exposure. However, the following hypothesis can be proposed. Sodium titanate nanotubes, nanofibers, and nanosheets were composed of sheets consisting of titanate. A larger area of the edge of the titanate sheet was exposed in nanofibers than in nanotubes and in nanosheets. Since the surface and the edge of the titanate sheet possessed different properties, as reported by Matsumoto et al. [28], the edge of the titanate sheet might become a chemically active site. The chemically active site had a specific effect on bacteria, and consequently a high antibacterial activity was expressed. We are currently attempting to elucidate the detailed mechanism underlying the antibacterial activity.

3.3. Antibacterial Activity of the Silver Nanoparticle/Silver Titanate Nanofiber Thin Film. The antibacterial activity of the silver nanoparticle/silver titanate nanofiber thin film against MRSA was investigated. The number of viable bacteria 


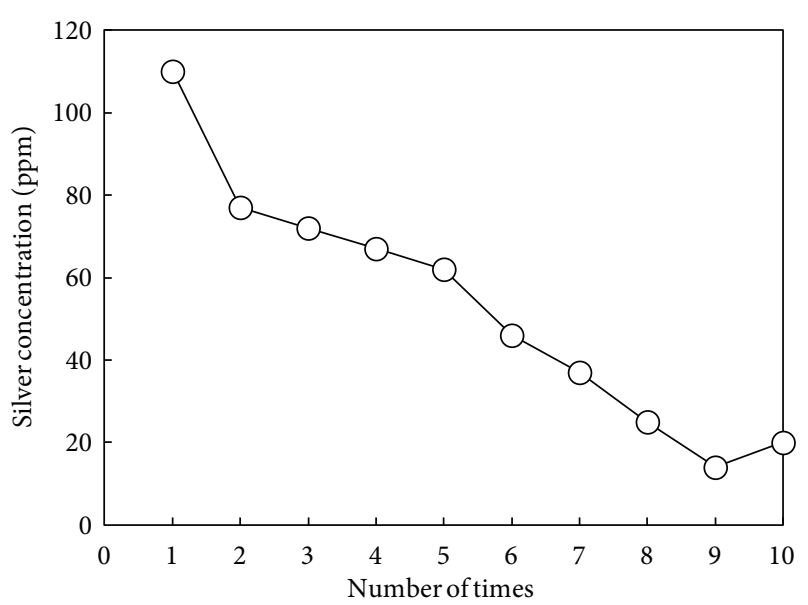

FIGURE 5: Repeated silver elution test performed on the silver nanoparticle/silver titanate nanofiber thin film.

immediately after inoculation of MRSA was $2.7 \times$ $10^{5} \mathrm{CFU} /$ sample. At $24 \mathrm{~h}$ after the inoculation, the number increased to $8.6 \times 10^{8} \mathrm{CFU} /$ sample on the blank sample, but decreased to below $1 \times 10^{1} \mathrm{CFU} /$ sample on the silver nanoparticle/silver titanate nanofiber thin film. Based on these values, the antibacterial activity value of the silver nanoparticle/silver titanate nanofiber thin film was calculated as 7.9, which indicated an extremely high antibacterial activity.

As shown in Figure 5, a large amount of silver (110 ppm) was eluted on the first day. Therefore, the elution of silver was suggested as a cause of the high antibacterial activity. However, the amount of eluted silver had decreased to $77 \mathrm{ppm}$ on the second day and this amount continued to decrease gradually over time, to $20 \mathrm{ppm}$ by the 10th day. This elution curve was similar to that reported previously by us for a silver nanoparticle/silver titanate nanotube thin film [24]. However, the amount of silver eluted on the first day was larger for the silver nanoparticle/silver titanate nanofiber thin film $(110 \mathrm{ppm})$ than for the previously reported silver nanoparticle/silver titanate nanotube thin film (98 ppm) [24]. The amount of eluted silver was considered to be greatly related to the amount of silver in the thin film. The amount of silver in the silver nanoparticle/silver titanate nanofiber thin film might differ from that in the silver nanoparticle/silver titanate nanotube thin film. However, since the amount of silver in nanofiber and nanotube thin films is extremely difficult to measure correctly and no effective measuring technique exists at present, the amount of silver in the thin films was not measured. Instead, we provide the minimum information on the amount of silver in these thin films by showing the results of EDX analysis of the thin film sample before the silver elution test. The molar ratio of $\mathrm{Ag} / \mathrm{Ti}$ for the silver nanoparticle/silver titanate nanofiber thin film was 0.638 , which was similar to 0.67 of that for the silver nanoparticle/silver titanate nanotube thin film [24]. For the actual amount of silver in the thin film, the nanostructure and the microstructure of the thin film including its density and thickness must be taken into consideration, in addition to the results of EDX analysis. The antibacterial activity value was also larger for the silver nanoparticle/silver titanate nanofiber thin film (7.9) than for the previous silver nanoparticle/silver titanate nanotube thin film (6.3). The reason for this difference was probably that a larger amount of silver was eluted from the silver nanoparticle/silver titanate nanofiber thin film.

The elution of silver from the silver nanoparticle/silver titanate nanofiber thin film is considered to be due to the elution from silver nanoparticle and the elution from silver titanate nanofiber caused by an ion exchange reaction due to high affinity between $\mathrm{Ag}^{+}$ions and proteins. The fetal bovine serum used as the solvent in the present study contains large amounts of proteins, which have very high affinity with $\mathrm{Ag}^{+}$ions through the $-\mathrm{SH}$ group or $-\mathrm{NH}$ group in the proteins, and appreciable concentrations of cations. Ion exchange reactions are generally known to proceed more rapidly than dissolution reactions of metals. Furthermore, we have already revealed that at the silver elution test of the silver nanoparticle/silver titanate nanotube thin film although silver at a concentration of tens of thousands of ppb was eluted in fetal bovine serum, silver at a concentration of only several hundreds of ppb could be eluted in physiological saline and PBS solutions [24]. Since the silver nanoparticle/silver titanate nanofiber reported in this study possessed a crystal structure that was basically similar to that of the silver nanoparticle/silver titanate nanotube, although the nanomorphology differed, the elution behavior of silver was presumed to be similar in both structures. Actually, as shown by the repeated silver elution test in Figure 5, the elution behavior of silver from the silver nanoparticle/silver titanate nanofiber thin film was similar to that from the silver nanoparticle/silver titanate nanotube thin film [24]. Therefore, the elution of silver from silver titanate nanofiber caused by the silver ion exchange reaction due to high affinity between $\mathrm{Ag}^{+}$ions and proteins was considered to contribute substantially to the large amount of eluted silver on the first day and the high antibacterial activity. When the elution time was extended, this contribution then would become smaller and the contribution of silver eluted from silver nanoparticles would become larger as time progressed.

\section{Conclusion}

The present study revealed that a sodium titanate nanofiber thin film and a silver nanoparticle/silver titanate nanofiber thin film formed on the surface of a titanium plate exhibited strong antibacterial activities against MRSA, which is one of the major bacteria causing in-hospital infections. The high antibacterial activity seen for the sodium titanate nanofiber thin film under UV irradiation indicated that bacteria could be prevented from growing and adhering to a sample when the sample was stored under UV irradiation. In contrast, the silver nanoparticle/silver titanate nanofiber thin film would be able to act efficiently as an antibacterial material in a living body where no light could penetrate. The titanate nanofiber thin film/titanium composites adhered firmly to titanium. 
Therefore, these composites will be extremely useful as implant materials that have excellent antibacterial activities.

\section{Acknowledgment}

This work was partially supported by KAKENHI (19750172) and Saga University Dean's Grant For Promising Young Researchers.

\section{References}

[1] H. M. Kim, F. Miyaji, T. Kokubo, and T. Nakamura, "Preparation of bioactive $\mathrm{Ti}$ and its alloys via simple chemical surface treatment," Journal of Biomedical Materials Research, vol. 32, no. 3, pp. 409-417, 1996.

[2] Z. R. Tian, J. A. Voigt, J. Liu, B. McKenzie, and H. Xu, "Large oriented arrays and continuous films of $\mathrm{TiO}_{2}$-based nanotubes," Journal of the American Chemical Society, vol. 125, no. 41, pp. 12384-12385, 2003.

[3] X. Peng and A. Chen, "Large-scale synthesis and characterization of $\mathrm{TiO}_{2}$-based nanostructures on Ti Substrates," Advanced Functional Materials, vol. 16, no. 10, pp. 1355-1362, 2006.

[4] W. Dong, T. Zhang, J. Epstein et al., "Multifunctional nanowire bioscaffolds on Titanium," Chemistry of Materials, vol. 19, no. 18, pp. 4454-4459, 2007.

[5] E. Hosono, H. Matsuda, I. Honma, M. Ichihara, and H. Zhou, "Synthesis of a perpendicular $\mathrm{TiO}_{2}$ nanosheet film with the superhydrophilic property without UV irradiation," Langmuir, vol. 23, no. 14, pp. 7447-7450, 2007.

[6] T. Kokubo, F. Miyaji, H. M. Kim, and T. Nakamura, "Spontaneous formation of bonelike apatite layer on chemically treated titanium metals," Journal of the American Ceramic Society, vol. 79, no. 4, pp. 1127-1129, 1996.

[7] H. M. Kim, F. Miyaji, T. Kokubo, and T. Nakamura, "Effect of heat treatment on apatite-forming ability of Ti metal induced by alkali treatment," Journal of Materials Science: Materials in Medicine, vol. 8, no. 6, pp. 341-347, 1997.

[8] H. M. Kim, F. Miyaji, T. Kokubo, S. Nishiguchi, and T. Nakamura, "Graded surface structure of bioactive titanium prepared by chemical treatment," Journal of Biomedical Materials Research, vol. 45, no. 2, pp. 100-107, 1999.

[9] S. Nishiguchi, T. Nakamura, M. Kobayashi, H. M. Kim, F. Miyaji, and T. Kokubo, "The effect of heat treatment on bonebonding ability of alkali-treated titanium," Biomaterials, vol. 20, no. 5, pp. 491-500, 1999.

[10] L. Jonášová, F. A. Müller, A. Helebrant, J. Strnad, and P. Greil, "Biomimetic apatite formation on chemically treated titanium," Biomaterials, vol. 25, no. 7-8, pp. 1187-1194, 2004.

[11] H. M. Kim, T. Himeno, M. Kawashita, J. H. Lee, T. Kokubo, and T. Nakamura, "Surface potential change in bioactive titanium metal during the process of apatite formation in simulated body fluid," Journal of Biomedical Materials Research. Part A, vol. 67, no. 4, pp. 1305-1309, 2003.

[12] S. Nishiguchi, S. Fujibayashi, H. M. Kim, T. Kokubo, and T. Nakamura, "Biology of alkali- and heat-treated titanium implants," Journal of Biomedical Materials Research. Part A, vol. 67, no. 1, pp. 26-35, 2003.

[13] K. Muramatsu, M. Uchida, H. M. Kim, A. Fujisawa, and T. Kokubo, "Thromboresistance of alkali- and heat-treated titanium metal formed with apatite," Journal of Biomedical Materials Research. Part A, vol. 65, no. 4, pp. 409-416, 2003.
[14] T. Kokubo and S. Yamaguchi, "Novel bioactive titanate layers formed on Ti metal and its alloys by chemical treatments," Materials, vol. 3, no. 1, pp. 48-63, 2010.

[15] X. J. Wang, Y. C. Li, J. G. Lin, Y. Yamada, P. D. Hodgson, and C. E. Wen, "In vitro bioactivity evaluation of titanium and niobium metals with different surface morphologies," Acta Biomaterialia, vol. 4, no. 5, pp. 1530-1535, 2008.

[16] L. Zhao, P. K. Chu, Y. Zhang, and Z. Wu, "Antibacterial coatings on titanium implants," Journal of Biomedical Materials Research. Part B, vol. 91, no. 1, pp. 470-480, 2009.

[17] K.-T. Oh, H. M. Shim, and K. N. Kim, "Properties of titaniumsilver alloys for dental application," Journal of Biomedical Materials Research. Part B, vol. 74, no. 1, pp. 649-658, 2005.

[18] W. Chen, Y. Liu, H. S. Courtney et al., "In vitro anti-bacterial and biological properties of magnetron co-sputtered silvercontaining hydroxyapatite coating," Biomaterials, vol. 27, no. 32, pp. 5512-5517, 2006.

[19] K. Das, S. Bose, A. Bandyopadhyay, B. Karandikar, and B. L. Gibbins, "Surface coatings for improvement of bone cell materials and antimicrobial activities of Ti implants," Journal of Biomedical Materials Research. Part B, vol. 87, no. 2, pp. 455-460, 2008.

[20] W.-H. Song, S. R. Hyun, and S. H. Hong, "Antibacterial properties of $\mathrm{Ag}$ ( or $\mathrm{Pt}$ )-containing calcium phosphate coatings formed by micro-arc oxidation," Journal of Biomedical Materials Research. Part A, vol. 88, no. 1, pp. 246-254, 2009.

[21] I. Noda, F. Miyaji, Y. Ando et al., "Development of novel thermal sprayed antibacterial coating and evaluation of release properties of silver ions," Journal of Biomedical Materials Research. Part B, vol. 89, no. 2, pp. 456-465, 2009.

[22] B. S. Necula, L. E. Fratila-Apachitei, S. A. J. Zaat, I. Apachitei, and J. Duszczyk, "In vitro antibacterial activity of porous $\mathrm{TiO}_{2}$ Ag composite layers against methicillin-resistant Staphylococcus aureus," Acta Biomaterialia, vol. 5, no. 9, pp. 3573-3580, 2009.

[23] M. Yada, Y. Inoue, M. Uota et al., "Plate, wire, mesh, microsphere, and microtube composed of sodium titanate nanotubes on a titanium metal template," Langmuir, vol. 23, no. 5, pp. 2815-2823, 2007.

[24] Y. Inoue, M. Uota, T. Torikai et al., "Antibacterial properties of nanostructured silver titanate thin films formed on a titanium plate," Journal of Biomedical Materials Research. Part A, vol. 92, no. 3, pp. 1171-1180, 2010.

[25] M. Yada, Y. Inoue, G. Akihito et al., "Apatite-forming ability of titanium compound nanotube thin films formed on a titanium metal plate in a simulated body fluid," Colloids and Surfaces B, vol. 80, no. 2, pp. 116-124, 2010.

[26] B. Chi, E. S. Victorio, and T. Jin, "Synthesis of $\mathrm{TiO}_{2}$-based nanotube on Ti substrate by hydrothermal treatment," Journal of Nanoscience and Nanotechnology, vol. 7, no. 2, pp. 668-672, 2007.

[27] X. Wang, Z. Jin, C. Feng, Z. Zhang, and H. Dang, "Alternative adsorption-desorption of $\mathrm{C}_{3} \mathrm{H}_{6}$ on nanotube-like silver titanate," Journal of Solid State Chemistry, vol. 178, no. 3, pp. 638-644, 2005.

[28] Y. Matsumoto, S. Ida, and T. Inoue, "Photodeposition of metal and metal oxide at the $\mathrm{TiO}_{\mathrm{x}}$ nanosheet to observe the photocatalytic active site," Journal of Physical Chemistry C, vol. 112, no. 31, pp. 11614-11616, 2008. 

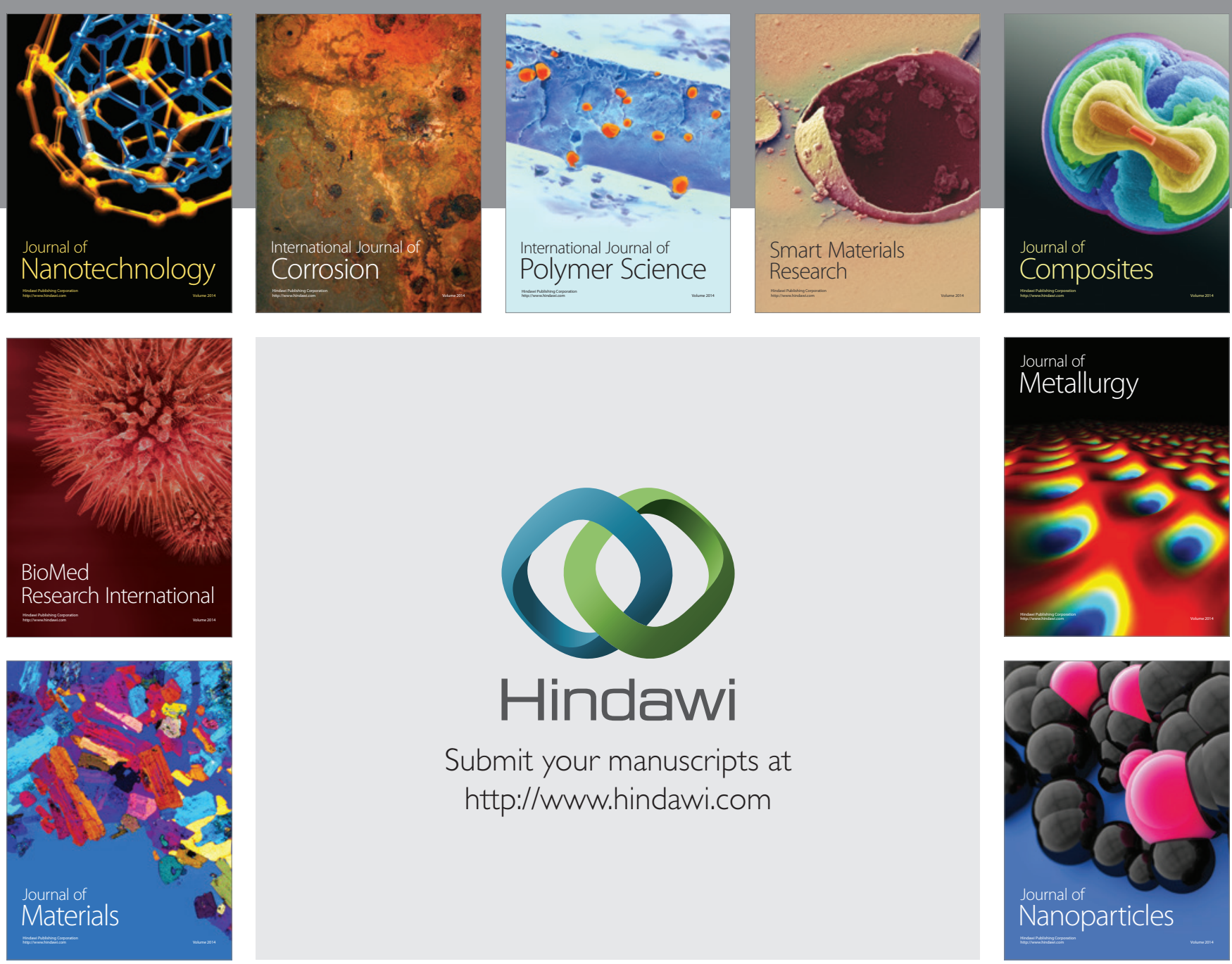

Submit your manuscripts at http://www.hindawi.com
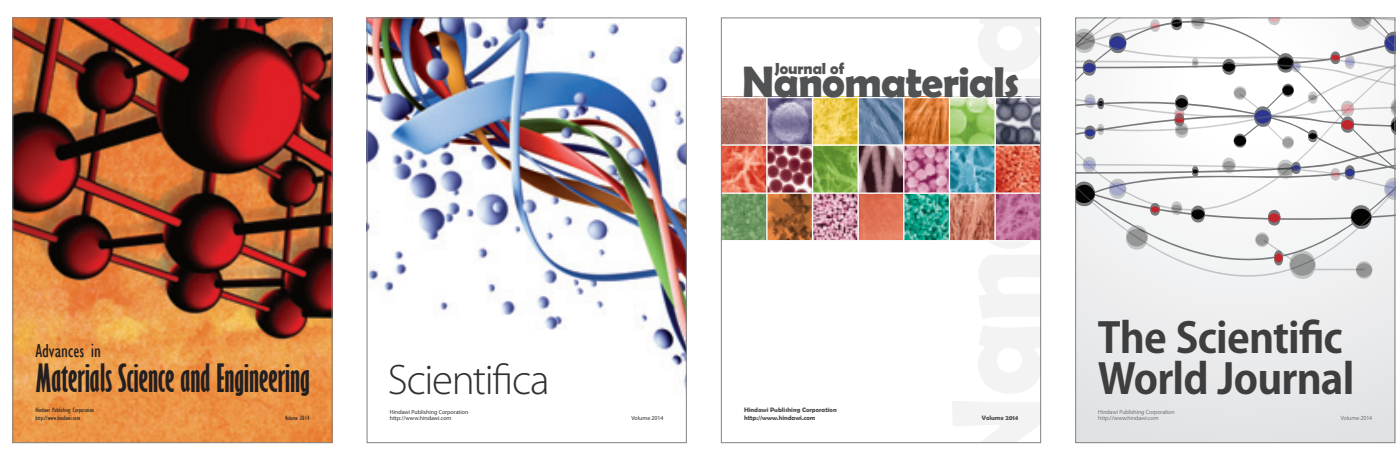

\section{The Scientific World Journal}
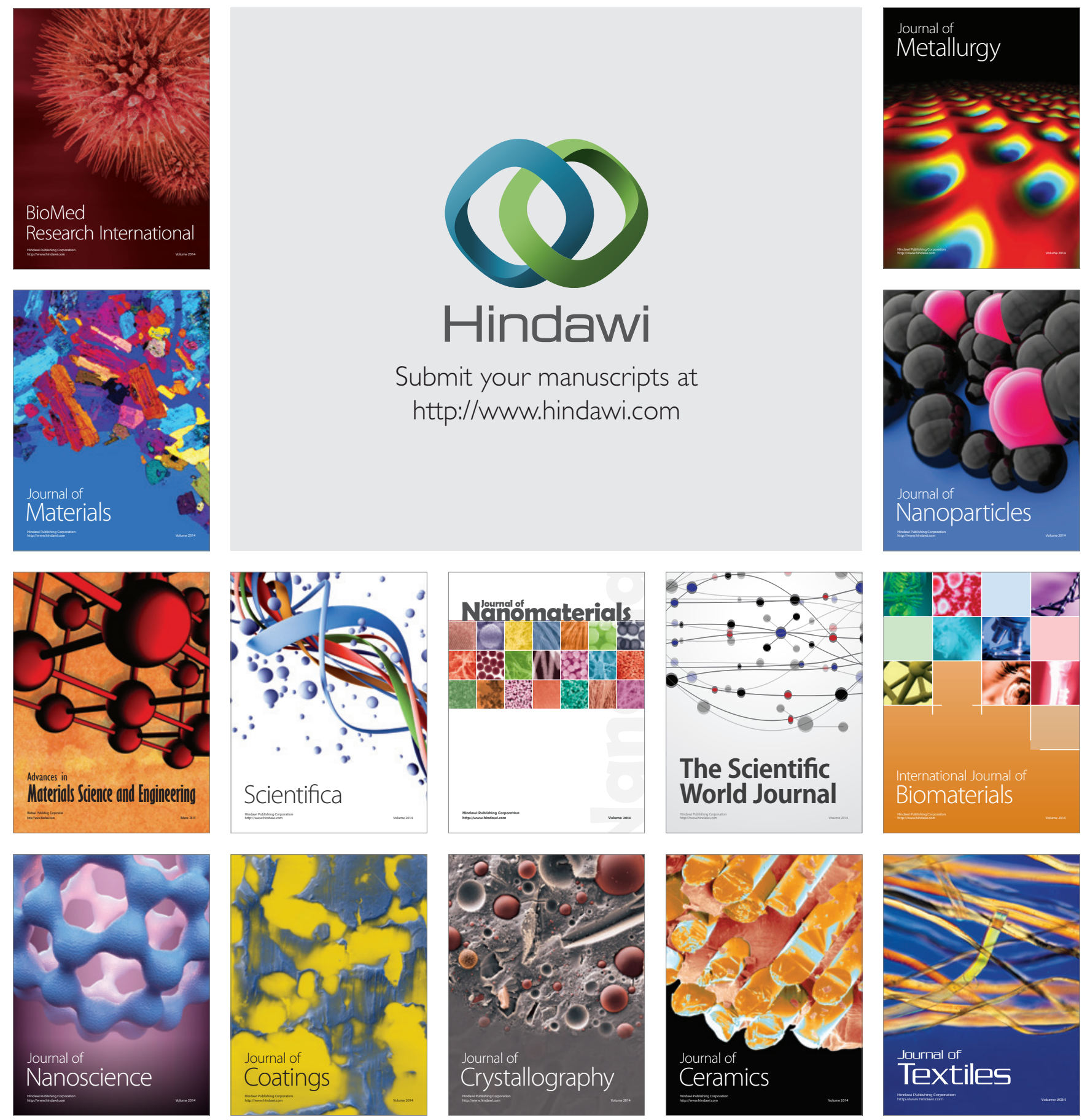